Pensamiento Crítico № 16, pp. 79-93

\title{
Curva de phillips y la tasa natural de desempleo
}

Investigador: Dr. Víctor Benigno Pérez Suárez

Alumno: Nieto Cueva Romel Eduardo

\section{RESUMEN}

Este trabajo trata de explicar, mediante una curva de Phillips (en un modelo simple bivariado), la relación que existe entre: inflación y tasa de desempleo en el caso peruano, además se estima la tasa natural de desempleo para una estimación de los últimos 19 años, aquí se muestra evidencia a favor del cumplimiento de la curva de Phillips. El análisis muestra evidencia empírica de la crítica de Lucas, debido a que la estimación no toma en cuenta los impactos de los cambios en la política económica y además al asumir expectativas adaptativas, los resultados se confrontan en las conclusiones. Además en el estudio se encuentra una relación negativa entre la tasa de desempleo y la variación de la tasa de inflación. Finalmente se manifiesta que la tasa de desempleo nacional está por debajo de la tasa natural de desempleo, mostrando alguna evidencia a favor de que pueda haber presiones inflacionarias.

Palabras clave: Curva de Phillips, Tasa natural de desempleo. 


\title{
Pensamiento Crítico $\mathrm{N}^{\circ}$ I6
}

\begin{abstract}
This paper tries to explain, through a Phillips curve (in a bivariate simple model), the relationship between: inflation and unemployment in the Peruvian case. In addition, it presents the natural rate of unemployment for the last 19 years, and shows evidence for the fulfillment of the Phillips curve. Empirical evidence of the Lucas' critique is shown; due to the fact that the estimate does not take into account the impacts of changes in the economic policy. The results are compared in the conclusions by assuming adaptive expectations. Moreover, the study displays a negative relationship between unemployment rate and the change in the inflation rate. Finally, it indicates that the national unemployment rate is below the natural rate of unemployment, which shows some evidence of possible inflationary pressures.
\end{abstract}

Keywords: Phillips curve, Natural rate of unemployment.

\section{La curva de Phillips original}

Alban William Housego Phillips (1914-1975), quien fuera profesor de economía, ciencia y estadística en la London School of Economics desarrolló una investigación empírica en 1958 en la que encontró una relación inversa y estable entre el desempleo y la tasa de cambio de los salarios monetarios, para el Reino Unido durante el período 1861-1957.

- Descubrió una relación negativa : $\quad \mathrm{Wt}=$ Constante- $\mathrm{lUt}$

- Altas tasas de paro llevaban asociadas bajas tasas de inflación

Paul Samuelson y Robert Solow mostraron dicha relación con datos de Estados Unidos durante el periodo 1900-1960.

- Observaron la misma relación inversa entre tasa de paro y tasa de inflación

- Bautizaron la relación como "curva de Phillips".

La existencia de una relación empírica fiable entre la tasa de paro y la tasa de inflación, unido a la existencia de una explicación teórica razonable, llevó a las 


\section{Víctor Benigno Pérez Suarez}

autoridades económicas a adoptar la curva de Phillips a la hora de tomar decisiones de política económica.

El objeto de la política macroeconómica de la década de los 60 en Estados Unidos tenía por finalidad mantener la tasa de paro en el intervalo que parecía coherente con una inflación moderada ( $\mathrm{pt}=\mathrm{C}$ - lut).

Sin embargo, a partir de 1970 deja de cumplirse dicha relación, en la década de 1970 se produce tanto una elevada inflación como un elevado desempleo asimismo subió el precio del petróleo desde 1970 esto incremento los costos no laborales, entonces las empresas subieron sus precios, por lo tanto los precios estuvieron permanentemente al alza. Durante la década de los 70 se produjeron dos fuertes incrementos del precio del petróleo que produjeron tanto una reducción de la producción (aumento de la tasa de paro) como un aumento de la tasa de inflación. La causa fundamental fue los cambios en la formación de expectativas de precios por parte de los trabajadores.

\section{Crítica de milton friedman y edmund phelps}

En general incluyen las expectativas de inflación y la explican como el hecho de que la inflación comienza a ser sistemáticamente positiva da lugar a que la inflación esperada por los agentes económicos ya no pueda ser nula, sino positiva. Además se hizo más persistente: si un año era alta, era más probable que en el siguiente periodo también lo fuera. Phelps ${ }^{1}$ y Friedman mostraron que la curva de Phillips no es estable porque la curva se desplaza cuando se cambien las expectativas de inflación.

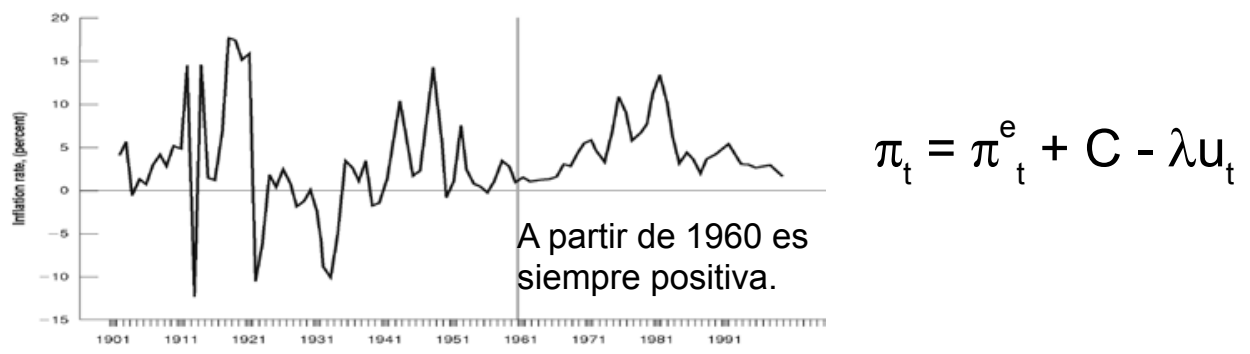

1 Phelps (1995) describe el proceso en este modo: “... el proceso de inflación creciente llevaría a la tasa de desempleo a chocar contra un nivel tal que los vendedores de dinero no encontrasen demandantes, dado que el dinero no jugaría ningún papel útil. El proceso de deflación creciente llevaría a la tasa de desempleo a un nivel tal que los vendedores de riqueza real argumentarían ser incapaces de encontrar compradores, pues el dinero ha venido a dominar todos los activos no monetarios, tales como bienes de consumo". 


\section{Pensamiento Crítico $\mathrm{N}^{\circ} 16$}

\section{Relación de oferta agregada}

Ahora lo que necesitamos es la función de oferta agregada inversa es decir no la relación de la producción con respecto a los precios sino de los precios con respecto a la producción, entonces tenemos ${ }^{2}$ :

$$
P_{t}=P_{t}^{e}+\theta\left(Y_{t}-\breve{y}\right)
$$

Donde $\mathrm{Y}_{\mathrm{t}}$ es el PBI real subyacente, mientras que $\breve{y}$, es el PBI real potencial; definimos el PBI potencial como el producto que puede generar una economía sin causar presiones inflacionarias.

Restamos Pt-1 a cada lado de la ecuación, y vamos a asumir que P es el logaritmo del nivel de precios.

$$
P_{t} / P_{t-1}=P_{t}^{e} / P_{t-1}+\theta\left(Y_{t}-\breve{y}\right)
$$

De donde tenemos:

$$
\pi_{t}=\pi_{t}^{e}+\theta\left(Y_{t}-\check{y}\right)
$$

Ahora aplicamos la Ley de Okun ${ }^{3}$. Especificamos la Ley de Okun con la siguiente ecuación:

$$
\theta\left(Y_{t}-\breve{y}\right)=-\psi\left(u_{t}-U_{n}\right)
$$

2 Esta representación de la oferta Agregada y la derivación del modelo se basa en el modelo de la curva de Phillips que se presenta en el Capitulo 13 de Mankiw (2005). Toda la derivación del modelo es propia del autor pero basada en las derivaciones de Mankiw (2005) y de Blanchard (2000), la ecuación final estimada econométricamente es una formulación que aparece en Blanchard (2000).

3 Esta relación fue propuesta por Arthur Okun(1962) 


\section{Víctor Benigno Pérez Suarez}

Y reemplazando en la ecuación anterior, tenemos:

$$
\pi_{t}=\pi_{t}^{e}-\Psi\left(u_{t}-U_{n}\right)
$$

Es una relación de la inflación con la inflación esperada y la desviación del desempleo con respecto a su tasa natural. Pero lo que queremos es una manera de hallar la NAIRU ${ }^{4}$, por eso es que despejamos de la siguiente forma:

$$
\pi_{t}=\pi_{t}^{e}+\Psi U_{n}-\Psi u_{t}
$$

Blanchard realiza la siguiente definición ${ }^{5}$ : “...La tasa de desempleo con la que las decisiones de precios y de salarios son coherentes se denomina tasa natural de desempleo. Esta terminología se ha convertido en la habitual, por lo que la adoptamos, pero el término "natural" es poco apropiado. Sugiere que se trata de una constante de la naturaleza, es decir, una constante a la que no le afectan las instituciones y la política económica".

Entonces si a " $\Psi \mathrm{U}_{\mathrm{n}}$ " lo tratamos como una constante y lo llamamos c, ya que $\Psi$ es una constante y Un permanece constante en el periodo de análisis, entonces al establecer $C=\psi$ Un obtenemos una expresión de la curva de Phillips como(*):

$$
\pi_{t}=C+\pi_{t}^{e}-\psi u_{t}
$$

$4 \quad$ Non-Accelerating Inflation Rate of Unemployment (NAIRU), según Mishkin (1998), fue inicialmente desarrollado por Modigliani y Papademos (1975). Se define como la tasa de desempleo que estabiliza la inflación en ausencia de sorpresas en la formación de precios y salarios.

5 Blanchard,O. Macroeconomía(1997) Prentice Hall Pág. 295. 


\section{Pensamiento Crítico $\mathrm{N}^{\circ} 16$}

Donde podemos decir que la inflación depende positivamente de la inflación esperada y negativamente del desempleo. Si suponemos expectativas adaptativas la inflación esperada será dependiente de la inflación del periodo anterior, así:

$$
\pi_{t}^{e}=\theta \pi_{t-1}
$$

Ahora veamos que pasa si $\theta$ toma algunos valores:

Si $\theta=0$ la curva de Phillips es la original

Si $\theta>0$ la inflación depende no solo de la tasa de desempleo sino también de la tasa de inflación del año pasado.

Si $\theta=1$, tenemos en $(*)$ :

$$
\pi_{t}=C+\pi_{t-1}-\psi u_{t}
$$

Es decir cuando $\theta=1$ la tasa de desempleo no afecta a la tasa de inflación sino a la variación de la tasa de inflación. A esta relación se le llama "Curva de Phillips modificada" o "Curva de Phillips con expectativas" o "Curva de Phillips aceleradora".

$$
\left(\pi_{t}-\pi_{t-1}\right)=C-\Psi u_{t}
$$

\section{La tasa natural de desempleo (tnd)}

Milton Friedman (1968) define la NAIRU como: "La tasa natural de desempleo es el nivel que sería fijado por el sistema walrasiano de ecuaciones de equilibrio general, con la condición de que se encuentren allí contempladas las características estructurales 


\section{Víctor Benigno Pérez Suarez}

actuales de los mercados de trabajo y bienes, y se incluyan las imperfecciones de mercado, la variabilidad estocástica de demandas y ofertas, el costo de recolección de información sobre vacantes y disponibilidades de mano de obra, los costos de la movilidad y otros".

Blanchard y Fisher (1989) asimilan la TND a la tasa media de desempleo y luego la asimilan a la tasa de desempleo de equilibrio. Hahn (1980) define la TND como el nivel de desempleo existente en pleno empleo. Clark y Summers (1979) sugieren que la TND es la tasa de desempleo "eficiente". Auerbach y Kotlikoff (1995) se refieren a la TND como "la más baja tasa de desempleo sostenible". Johnson y Layard (1986) manejan una analogía entre la tasa media de desempleo, la tasa de desempleo de equilibrio y lo que Friedman denominó la TND. Hall y Lilien (1986) relacionan la TND con la tasa de desempleo normal resultante de una percepción correcta de los niveles y cambios de precios y salarios por parte de trabajadores y firmas. Lilien (1982) había definido previamente la TND como la tasa de desempleo friccional. Mankiw (1994) define la TND como la tasa de desempleo del estado estable de la economía. Finalmente, Christiano (1997) define la TND como el componente tendencial del desempleo estimado por el filtro de Hodrick-Prescott.

Rogerson(1997) escribe un artículo en el que identifica las diez interpretaciones diferentes mencionadas en el párrafo anterior al término TND y concluye que un concepto que lleva a interpretaciones tan ambiguas esta lejos de ser útil en una investigación metódica. Simplemente, al no existir consenso sobre el elemento a estimar, no puede existir un cuerpo analítico coherente, en términos de comparación de estimaciones y discusión común sobre las implicaciones de política económica ${ }^{6}$.

En Perú, Félix Jiménez define el desempleo natural como “...un desempleo consistente con el equilibrio del mercado de trabajo y el producto de pleno empleo, al porcentaje de este tipo de desempleados se le denomina tasa natural de desempleo". Además nos dice que la TND es equivalente al desempleo friccional que surge como resultado de las propias fricciones del mercado de trabajo ${ }^{7}$.

$6 \quad$ Hector Javier Fuentes López, Favian Andrés Rodríguez Buitrago, "La Nairu": una aproximación teórica (1a parte)-Apuntes del CENES II semestre del 2007- Pág. 120-121.

7 Félix Jiménez, Macroeconomía Keynesiana de la Determinación de los Niveles de Producción y EmpleoMayo 1999 PUCP, pág.6. 


\section{Pensamiento Crítico $\mathrm{N}^{\circ} 16$}

Por definición la NAIRU es la tasa de desempleo con la que el nivel efectivo de precios es igual al esperado, es decir: $\pi \mathrm{t}=\pi_{\mathrm{t}}^{e}$ Entonces representando la NAIRU como un, $y$ ya que $\pi_{t}=\pi_{t}^{e}=\pi_{t-1}$

$\begin{array}{ll}\text { Tendremos: } & \pi \mathrm{t}-\pi_{\mathrm{t}-1}=0 \\ \text { Entonces: } & \left(\pi \mathrm{t}-\pi_{\mathrm{t}-1}\right)=0=\mathrm{C}+\beta \mathrm{u}_{\mathrm{t}} \\ \text { Y luego: } & \mathrm{Un}=\mathrm{C} / \beta\end{array}$

Estimación del Modelo para la Economía Peruana (data anual: 1993-2011)

$$
\text { Modelo: }\left(\pi_{t}-\pi_{t-1}\right)=C-\beta u_{t}
$$

El que la pendiente de la curva de Phillips dependa del régimen de política macroeconómica esta en la base de lo que hoy se conoce como la critica de Lucas ${ }^{8}$. Este plantea que usar modelos sin especificar la estructura de la economía dada por sus políticas macroeconómicas generaría coeficientes no significativos, la razón es que los parámetros del modelo dependen de las políticas, las cuales no se incluyen en este trabajo; ergo se muestran que los coeficientes de nuestro modelo calculado son no significativos, sin embargo el modelo es valido para nuestro análisis luego de hacerle las pruebas econométricas requeridas 9 .

\begin{tabular}{|c|c|c|c|c|}
\hline \multicolumn{5}{|c|}{$\begin{array}{l}\text { Dependent Variable: I } \\
\text { Method: Least Squares } \\
\text { Date: 06/21/12 Time: } 09: 34 \\
\text { Sample (adjusted): } 19952011 \\
\text { Included observations: } 17 \text { after adjustments } \\
\text { Convergence achieved after } 6 \text { iterations } \\
\end{array}$} \\
\hline Variable & Coef $\square$ cient & Std $\square \square$ rror & $\mathrm{t} /$ Statistic & $\square$ rob $\square$ \\
\hline $\mathrm{C}$ & $11 \square 5 \square \square 45$ & $7 \square 132905$ & $1 \square 624647$ & $0 \square 1265$ \\
\hline $\mathrm{D} \square \mathrm{S} \square \mathrm{M} \square \mathrm{L} \square \square$ & $\square 1476016$ & $0 \square \square 69751$ & $\square 1697056$ & $0111 \square$ \\
\hline$\square \square(1)$ & $0 \square 39560 \square$ & $0 \square 10714$ & $3 \square 693342$ & $0 \square 0024$ \\
\hline$\square$ squared & $0 \square 507449$ & & $\square 1195902$ \\
\hline$\square$ djusted $\square$ squared & $0 \square 4370 \square 5$ & \multicolumn{2}{|c|}{$\mathrm{S} \square \mathrm{D} \square$ dependent va } & $3 \square 59031 \square$ \\
\hline$S \square \square \square$ of regression & $2 \square 693732$ & \multirow{2}{*}{\multicolumn{2}{|c|}{$\begin{array}{l}\square \square \text { ai } \square \text { e info criterion } \\
\text { Sch } \square \text { ar } \square \text { criterion }\end{array}$}} & $4 \square 97 \square 51 \square$ \\
\hline Sum squared resid & $101 \square 5 \square 67$ & & & $5 \square 125555$ \\
\hline Log li $\square$ elihood & $\square \square 31740$ & \multirow{2}{*}{\multicolumn{2}{|c|}{$\begin{array}{l}\square \text { annan } \square \text { uinn criter } \\
\text { Durbin } \square \text { atson stat }\end{array}$}} & $4 \square 993134$ \\
\hline statistic & $7 \square 21730$ & & & $2 \square 576969$ \\
\hline$\square \operatorname{rob}(\square$ statistic) & $0 \square 007033$ & \multicolumn{2}{|c|}{ Durbin $\square$ atson stat } & \\
\hline
\end{tabular}

8 Mayor referencia sobre la crítica y el modelo de Lucas en: José De Gregorio, Macroeconomía Intermedia(2004) cáp. 21

$9 \quad$ Pruebas en el anexo 1 


$$
\begin{gathered}
\left(\pi_{t}-\pi_{t-1}\right)=11.5884-1.476 u_{t}+V_{t} \\
U_{n}=C / \beta=11.58845 / 1.476016 \\
U_{n}=7.8511
\end{gathered}
$$

Tendremos entonces para nuestro periodo de análisis bajo los supuestos establecidos que la tasa natural de desempleo anual peruana será de $7.85 \%$.

Como se menciono anteriormente, según Christiano ${ }^{10}$ (1997) define la TND como el componente tendencial del desempleo estimado por el filtro de Hodrick-Prescott, el cual calculamos para una data mensual en miles de personas desocupadas en Lima de marzo del 2001 a abril del $2012^{11}$.

Primero separamos el componente estacional del no estacional de la variable desempleo y calculamos el filtro de Hodrick-Prescott ${ }^{12}$, obteniendo:
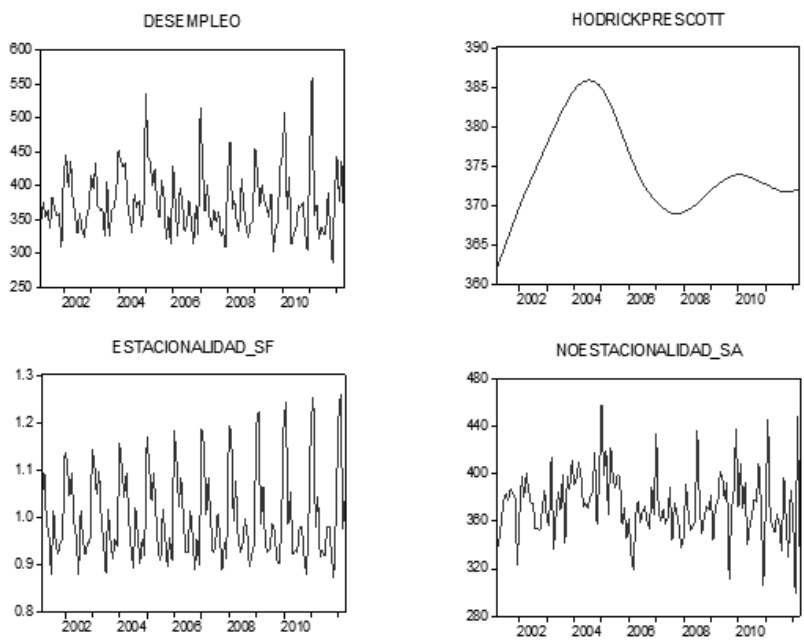

10 A pesar de que Rogerson no presenta una referencia bibliográfica de la definición de Christiano, aduciendo que le fue comentada en una conversación privada, una referencia bibliográfica al respecto puede encontrarse en Henao y Rojas (1998), que en su estimación de la TND al utilizar el filtro de Hodrick \& Prescott, citan a Pichelmann y Ulrich (1997).

11 Fuente: Estadísticas del Banco Central De Reserva del Perú

12 Se adjunta la programación en el anexo 2 


\section{Pensamiento Crítico $\mathrm{N}^{\circ} 16$}

Luego, observamos por el componente tendencial del filtro de Hodrick-Prescott una tendencia decreciente para el desempleo de Lima en el caso peruano; mostramos a su vez otros gráficos de análisis:

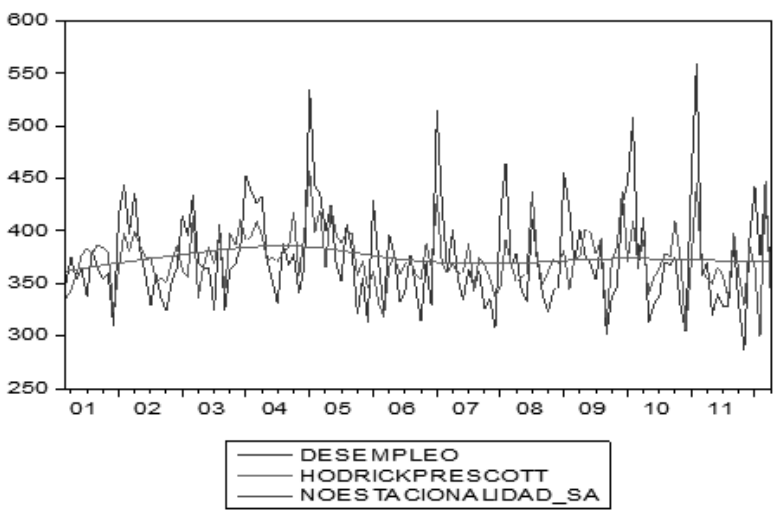

$\mathrm{Al}$ analizar gráficamente la serie, luego de quitarle la estacionalidad y la tendencia podemos observar el ciclo que mantiene el desempleo de Lima en el caso peruano.

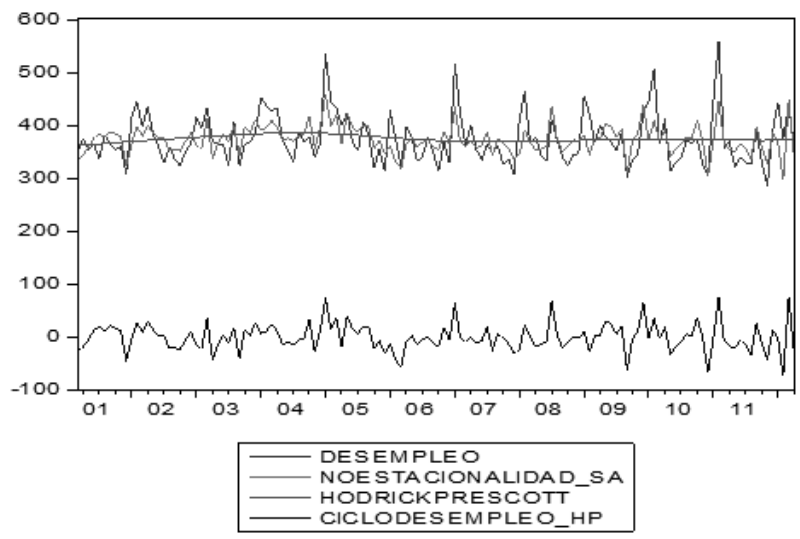

Obteniendo la media aritmética del componente tendencial calculado por el filtro de Hodrick-Prescott tendremos el promedio de la tasa natural de desempleo el cual es 374.51871, para nuestro análisis; en miles de personas desempleadas en Lima. 


\section{Víctor Benigno Pérez Suarez}

\section{CONCLUSIONES}

- Se puede concluir que la pendiente de la curva de Phillips depende de las características de la economía con pendiente negativa. En una economía con mucha volatilidad monetaria donde la sensibilidad de los salarios es alta, la curva de Phillips tenderá a ser vertical en el largo plazo. En cambio en una economía monetariamente estable, los cambios en la sensibilidad de los salarios serán percibidos principalmente como cambios de precios relativos y por lo tanto la curva de oferta será más horizontal.

- Entonces diremos que cuando la tasa de desempleo sea $7.851 \%$. Es por lo tanto esta tasa de desempleo la que mantiene la tasa de inflación estable, invariable.

- Cuando la tasa de desempleo sea $7.851 \%$ la variación de la inflación será cero pero los precios crecerán a la tasa $\pi_{t}=\pi_{t-1}$.

- En el 2011 el desempleo anual fue de 6.7\%, el cual al ser menor a nuestra estimación de la tasa natural de desempleo, nos muestra evidencia a favor de la existencia de presiones inflacionarias vistas en el 2011 donde se observo un nivel de inflación mayor al rango meta de $2 \%+/-1 \%$ para el caso peruano.

- El filtro de Hodrick-Prescott nos muestra un comportamiento tendencial decreciente para el desempleo mensual peruano en los últimos once años.

- El promedio de la tasa natural de desempleo mensual estimada es 374.51871, mayor al promedio de desempleo anual del año 2011, el cual fue de 370.40910, en miles de desempleados en la ciudad de Lima. 


\section{Pensamiento Crítico $\mathrm{N}^{\circ} 16$}

\section{ANEXO 1}

Programa y pruebas para el modelo de Economía Peruana (data anual: 1993-2011)

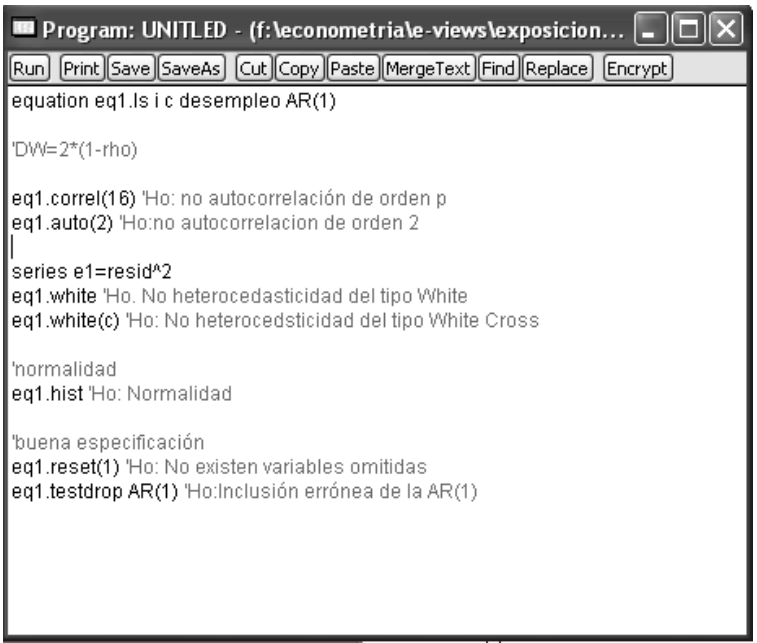

\begin{tabular}{|c|c|c|c|c|c|c|c|c|}
\hline \multicolumn{8}{|c|}{$\square$ Equation: EQ1 Workfile: UNTITLED::UntitledI } & $\square \square$ \\
\hline \multicolumn{9}{|c|}{\begin{tabular}{|llll} 
View Proc Object Print Name Freeze Estimate Forecast Stats Resids \\
\end{tabular}} \\
\hline \multicolumn{9}{|c|}{ Correlogram of Residuals } \\
\hline \multicolumn{9}{|c|}{$\begin{array}{l}\text { Date: } 07 / 03 / 12 \text { Time: } 02: 40 \\
\text { Sample: } 19952011 \\
\text { Included observations: } 17 \\
\text { Q-statistic probabilities adjusted for } 1 \text { ARMA term(s) }\end{array}$} \\
\hline \multicolumn{2}{|c|}{ Autocorrelation } & Partial Cor & lation & & $\mathrm{AC}$ & PAC & Q-Stat & Prob \\
\hline $1 \square$ & 1 & $1 \square$ & 1 & & -0.328 & -0.328 & 2.1752 & \\
\hline 1 & 1 & 1 口 & 1 & & -0.031 & -0.156 & 2.1964 & 0.138 \\
\hline 10 & 1 & 1 且 & 1 & & -0.097 & -0.183 & 2.4116 & 0.299 \\
\hline & 1 & & 1 & & 0.143 & 0.043 & 2.9162 & 0.405 \\
\hline 15 & 1 & ] & 1 & & -0.137 & -0.110 & 3.4244 & 0.489 \\
\hline 1 & 1 & $1 \square$ & 1 & & -0.239 & -0.379 & 5.0973 & 0.404 \\
\hline 1 & 1 & 10 & 1 & 7 & 0.171 & -0.098 & 6.0389 & 0.419 \\
\hline 1 & 1 & 马 & 1 & 8 & 0.160 & 0.122 & 6.9612 & 0.433 \\
\hline $1 \square$ & 1 & $1 \square$ & 1 & & -0.268 & -0.277 & 9.8677 & 0.274 \\
\hline 1 ב & 1 & 1 & 1 & 10 & 0.131 & -0.007 & 10.663 & 0.299 \\
\hline$\sqrt{5}$ & 1 & 마 & 1 & 11 & -0.066 & -0.173 & 10.897 & 0.366 \\
\hline & 1 & 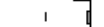 & 1 & 12 & 0.194 & -0.030 & 13.330 & 0.272 \\
\hline 16 & 1 & 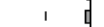 & 1 & 13 & -0.226 & -0.063 & 17.472 & 0.133 \\
\hline 10 & 1 & 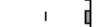 & 1 & 14 & 0.086 & -0.056 & 18.260 & 0.148 \\
\hline 1 & 1 & C & 1 & 15 & 0.024 & -0.141 & 18.350 & 0.191 \\
\hline 1 & 1 & 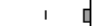 & 1 & 16 & -0.015 & -0.073 & 18.424 & $\begin{array}{ll}4 & 0.241\end{array}$ \\
\hline
\end{tabular}

No Heterocedasticidad: Se acepta la Hipótesis Nula 


\section{Víctor Benigno Pérez Suarez}

\begin{tabular}{|c|c|c|c|c|}
\hline$\square$ Equation: EQ01 W & orkfile: UN7 & TITLED::Untit| & & $-\square \times$ \\
\hline View Proc Object Print N & ame Freeze & Estimate Foreca & Stats Resids & \\
\hline Heteroskedasticity Test: & White & & & \\
\hline F-statistic & 1.940282 & Prob. $F(5,11)$ & & 0.1670 \\
\hline Obs*R-squared & 7.966799 & Prob. Chi-Sq & $\operatorname{are}(5)$ & 0.1581 \\
\hline Scaled explained SS & 3.576625 & Prob. Chi-Sq & $\operatorname{are}(5)$ & 0.6118 \\
\hline Test Equation: & & & & \\
\hline Dependent Variable: RES & $D^{n} 2$ & & & \\
\hline Method: Least Squares & & & & \\
\hline Date: $07 / 03 / 12$ Time: 02 & & & & \\
\hline Sample: 19952011 & & & & \\
\hline Included observations: 1 & & & & \\
\hline Collinear test regressors & dropped frol & m specification & & \\
\hline Variable & Coefficient & Std. Error & t-Statistic & Prob. \\
\hline $\mathrm{C}$ & 72.73150 & 58.89481 & 1.234939 & 0.2426 \\
\hline GRADF_01*GRADF_02 & -56.13289 & 40.35411 & -1.391008 & 0.1917 \\
\hline GRADF_01*GRADF_03 & -4.679261 & 4.421945 & -1.058191 & 0.3127 \\
\hline คDA & A กก1989 & ว AQAAan & 1 Б1ก7A & ก1255 $v$ \\
\hline
\end{tabular}

Test de Normalidad: Se acepta la Hipótesis Nula

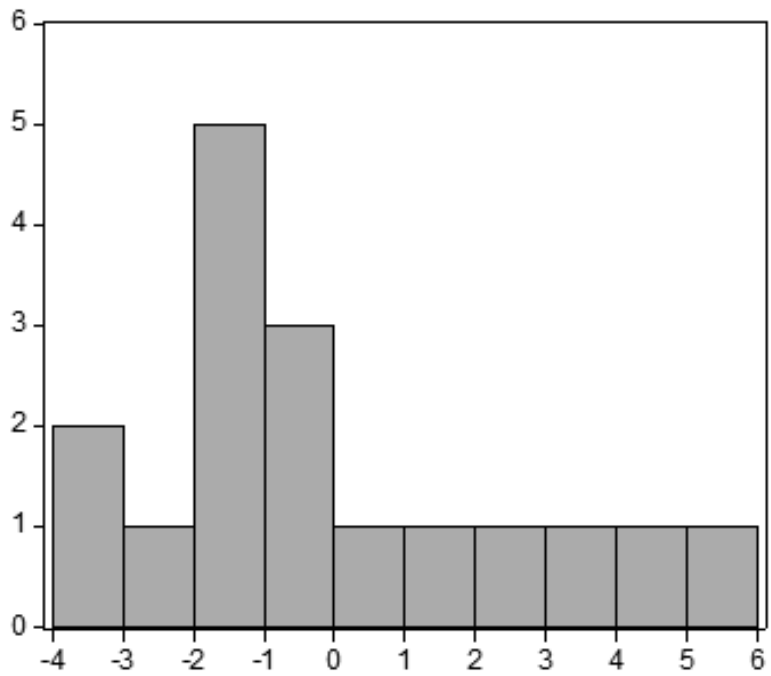

\begin{tabular}{lc|}
\hline \multicolumn{2}{|l|}{ Series: Residuals } \\
Sample 1995 2011 \\
Observations 17 \\
Mean & $6.52 \mathrm{e}-14$ \\
Median & -0.378360 \\
Maximum & 5.052164 \\
Minimum & -3.515916 \\
Std. Dev. & 2.519756 \\
Skewness & 0.523959 \\
Kurtosis & 2.323919 \\
& \\
Jarque-Bera & 1.101612 \\
Probability & 0.576485 \\
\hline
\end{tabular}




\section{Pensamiento Crítico $\mathrm{N}^{\circ} 16$}

Se acepta la inclusión del $\mathrm{AR}(1)$, al rechazarse la Hipótesis Nula de inclusión errónea del AR(1).

\begin{tabular}{|c|c|c|c|c|}
\hline \multicolumn{4}{|c|}{$\square$ Equation: EQ1 Workfile: UNTITLED::UntitledI } & - \\
\hline \multicolumn{5}{|c|}{ Yiew Proc Object] [Print] Name Freeze [Estimate Forecast] Stats] Resids] } \\
\hline \multicolumn{5}{|c|}{ Redundant Variables: AR(1) } \\
\hline F-statistic & 13.66503 & \multirow{2}{*}{\multicolumn{2}{|c|}{$\begin{array}{l}\text { Prob. F(1,14) } \\
\text { Prob. Chi-Square(1) }\end{array}$}} & 0.0024 \\
\hline Log likelihood ratio & 11.57890 & & & 0.0007 \\
\hline \multicolumn{5}{|c|}{$\begin{array}{l}\text { Test Equation: } \\
\text { Dependent Variable: I } \\
\text { Method: Least Squares } \\
\text { Date: } 07 / 03 / 12 \text { Time: } 02: 44 \\
\text { Sample: } 1995 \text { 2011 } \\
\text { Included observations: } 17\end{array}$} \\
\hline Variable & Coefficient & Std. Error & $\mathrm{t}$-Statistic & Prob. \\
\hline $\begin{array}{c}\mathrm{C} \\
\text { DESEMPLEO }\end{array}$ & $\begin{array}{r}4.175288 \\
-0.667668\end{array}$ & $\begin{array}{l}8.422764 \\
1.041169\end{array}$ & $\begin{array}{r}0.495715 \\
-0.641267\end{array}$ & $\begin{array}{l}0.6273 \\
0.5310\end{array}$ \\
\hline R-squared & 0.026683 & \multicolumn{2}{|c|}{ Mean dependent var } & -1.195902 \\
\hline Adjusted R-squared & -0.038204 & \multicolumn{2}{|c|}{ S.D. dependent var } & 3.590318 \\
\hline S.E. of regression & 3.658258 & \multirow{2}{*}{\multicolumn{2}{|c|}{$\begin{array}{l}\text { Akaike info criterion } \\
\text { schwarz criterion }\end{array}$}} & 5.541982 \\
\hline Sum squared resid & 200.7428 & & & 5.640008 \\
\hline Log likelihood & -45.10685 & \multicolumn{2}{|c|}{$\begin{array}{l}\text { Schwarz criterion } \\
\text { Hannan-Quinn criter. }\end{array}$} & 5.551726 \\
\hline F-statistic & 0.411224 & \multicolumn{2}{|c|}{ Durbin-Watson stat } & 1.417111 \\
\hline Prob(F-statistic) & 0.531026 & & & \\
\hline
\end{tabular}

\section{ANEXO 2}

Programa para cálculo del filtro de Hodrick-Prescott, utilizado en el presente trabajo.

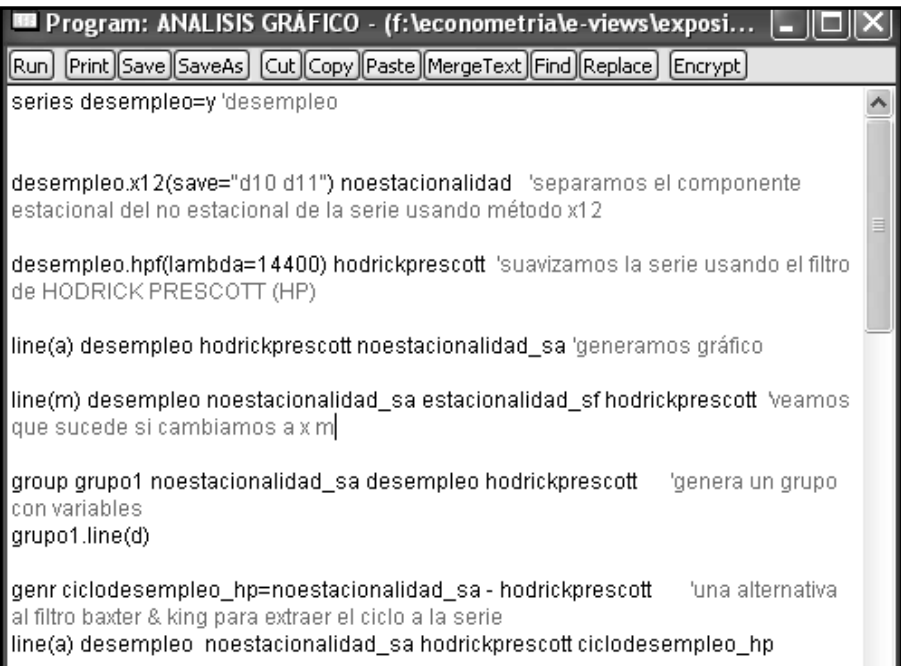




\section{Víctor Benigno Pérez Suarez}

\section{Bibliografía}

José De Gregorio, Macroeconomía Intermedia (2004) cáp.21.

Richard Froyen, Macroeconomía, Teoría y Políticas 5ta. Edición, cáp.10.

Blanchard,O. Macroeconomía(1997) Prentice Hall

Yadira Díaz, Juan Carlos Guataquí, Javier Alberto Gutiérrez, La Tasa De Desempleo en Bogotá: teoría y evidencia empírica (abril de 2001), trabajo presentado al seminario del 28 de marzo de 2001 del Banco de la República de Colombia.

Héctor Javier Fuentes López, Favian Andrés Rodríguez Buitrago, "La Nairu": una aproximación teórica (1a parte)-Apuntes del CENES II semestre del 2007

Eduardo Salazar Silva, Curva de Phillips y la Tasa Natural de Desempleo. Una aproximación simple para el Perú. (1993 - 2006)- Versión: 01 de Enero del 2008-Universidad Nacional Pedro Ruiz Gallo y Grupo de Investigación Económica.

Juan Carlos Guataquí Roa, Estimaciones de la Tasa Natural de Desempleo en Colombia, No. 2 Enero 2000 Universidad del Rosario 